\title{
Multiwavelength Fiber Sources Based on Double-Pass Superfluorescent Fiber Sources
}

\author{
C. D. Su and Lon A. Wang, Associate Member, IEEE
}

\begin{abstract}
We demonstrate a new technique to generate a high-power multiwavelength fiber source (MWFS) by inserting cascaded long-period fiber gratings (LPFG's) in double-pass Er-doped fiber (EDF) superfluorescent fiber sources. 20 channels with extinction ratios larger than $10 \mathrm{~dB}$ can be obtained between $1525-1560 \mathrm{~nm}$ with appropriate EDF lengths. Though the total output power decreases due to the insertion of the cascaded LPFG's, the power in each channel for the proposed MWFS is larger than that obtained by using the spectrum slicing technique because of power redistribution. We also demonstrate that by using another LPFG in such a source to reduce the power variation among the desired channels. The wavelength stability of the source is shown to be determined by the cascaded LPFG's rather than by the EDF.
\end{abstract}

Index Terms-Fiber source, long-period fiber grating (LPFG).

\section{INTRODUCTION}

W AVELENGTH-DIVISION multiplexing (WDM) is an important technique for fiber optical communication since it can increase transmission capability by orders of magnitude. A multiwavelength light source is always desired in a WDM system. A multiple number of lasers operating at different wavelengths have been reported [1], [2], however, the lasers should be fabricated and operated at specific wavelengths, which may increase cost and complexity. Recently, a multiwavelength fiber source (MWFS) employing "spectrum slicing technique" [3]-[7] have attracted much attention because of their all-fiber configuration and relatively easy implementation. A filter with multipeak spectrum is placed at the output end of a broadband light source to slice the spectrum into many channels. Since only one broadband light source is needed for the spectrum sliced fiber source, the issues of cost and complexity may be relieved.

Several broadband light sources such as light emitting diodes (LED's) [3], superluminescent laser diodes (SLD's) [4], and Er-doped superfluorescent fiber sources (SFS's) [5] have been used for spectrum sliced fiber sources. Although a light source with broader spectrum could generate more channels, large output power and high wavelength stability of the source is also required. The bandwidth of a LED or a SLD could be larger

Manuscript received October 6, 1999; revised January 6, 2000. This work was supported in part by the National Science Council, Taiwan, R.O.C., under Contract NSC 89-2215-E-002-013.

C. D. Su is with the Department of Electrical Engineering and Institute of Electro-Optical Engineering National Taiwan University Taipei, Taiwan, R.O.C.

L. A. Wang is with the Department of Electrical Engineering and Institute of Electro-Optical Engineering National Taiwan University Taipei, Taiwan, R.O.C. $\mathrm{He}$ is also with the Department of Electrical Engineering, National Taiwan University, Taipei, Taiwan, R.O.C. (e-mail: lon@ccms.ntu.edu.tw).

Publisher Item Identifier S 0733-8724(00)03742-7. than $100 \mathrm{~nm}$, however, its applications for WDM systems may be limited due to low output power. Although the bandwidth of an Er-doped SFS, about $40-50 \mathrm{~nm}$, is smaller than that of a LED or a SLD, the output power is one to two orders of magnitude larger [8]. Therefore, it has been widely used as the light source for spectrum sliced source in the past years. Fabry-Perot filters [6] and arrayed-waveguide gratings [7] have been used as the filter in the spectrum sliced fiber source. In [9], cascaded LPFG's are used as the filter for the spectrum sliced fiber source. Since the filter is placed at the output end of a broadband light source for spectrum sliced fiber source, it incurs a marked power loss. To reduce such a power loss, one could introduce a mechanism to cause power redistribution from the unwanted wavelength range to the wanted wavelength range. We have realized this idea by inserting cascaded LPFG's into a conventional double-pass backward (DPB) SFS [10], and the results show that output power of each channel is larger than that of the spectrum sliced fiber source.

In this paper, we demonstrate a MWFS with larger power for each channel by adding cascaded LPFG's inside a SFS in both double-pass backward and forward (DPF) configurations. The cascaded LPFG's in the proposed configurations not only provide the filtering effect but also cause power redistribution. Thus, the output power of such a MWFS is larger than the spectrum sliced source. The rest of the paper is arranged as follows. In Section II, the characteristics of the MWFS's based on both DPB and DPF configurations are shown. In Section III, we propose a technique by inserting another LPFG to reduce the power variation among channels. The temperature effects of EDF and cascaded LPFG's are discussed. Finally, we conclude our study in Section IV.

\section{EXPERIMENT SETUP AND RESULTS}

Fig. 1(a) and (b) shows the setup of a MWFS based on DPB and DPF configurations, respectively. The output signal is in the same direction with the pump signal for the forward configuration and in the opposite direction for the backward configuration. The double-pass configurations are employed due to the higher output power than the single-pass ones [8], [11]. The pump power is launched through a WDM into a section of Er-doped fiber (Fibercore DF1500F-980) from a diode laser operating at $976 \mathrm{~nm}$. For the DPB configuration, another WDM is used to separate the residual pump signal and the forward signal, and to avoid the feedback of pump power to the pump laser. A fiber mirror, which provides the double-pass function, is used to reflect the signal to the opposite direction and propagate through the EDF again. The cascaded LPFG's are positioned between 


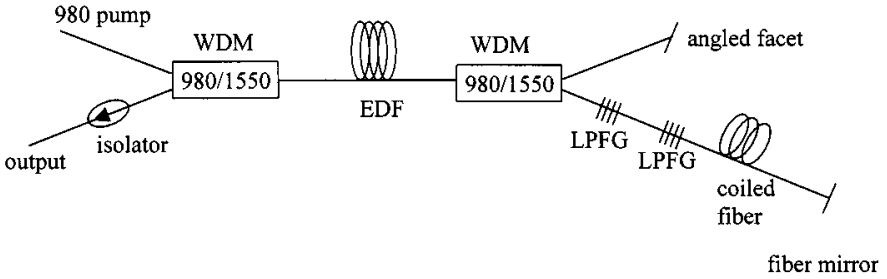

(a)

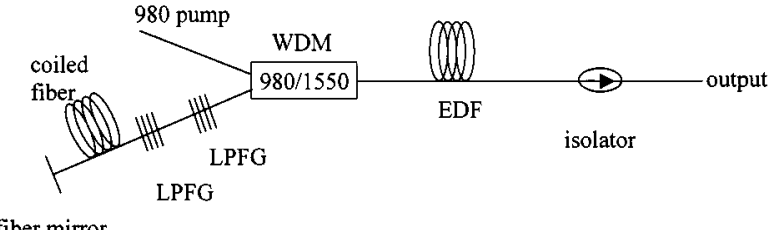

(b)

Fig. 1. Schematic diagram of a multiwavelength fiber source based on (a) DPB and (b) DPF configurations.

the fiber mirror and WDM to provide the filtering effect. An isolator with $\sim 60 \mathrm{~dB}$ isolation is used to prevent the formation of resonant cavity due to the optical feedback from the output endface. An optical spectrum analyzer with wavelength resolution of $0.07 \mathrm{~nm}$ is used for spectrum measurement.

The LPFG's are externally written in a hydrogenated dispersion shifted fiber by using a $\mathrm{KrF}$ excimer laser. After exposure, a periodic index variation is formed in the core. The two LPFG's are controlled to have the same transmission spectra at the same exposure dosage. The periodic index variation causes light to be coupled from the core mode to the cladding mode when the phase matching condition is satisfied [12]. The cascaded LPFG's act like a Mach-Zehnder interferometer. A LPFG acts like a beam splitter since it can couple the core mode to the cladding mode and vice versa. Due to the different refractive indices between core and cladding, an optical path difference results after the two modes propagate the same distance between the LPFG's. As the cladding mode is coupled back to the core mode by the second LPFG, it will interfere with the original core mode and lead to an interference pattern. The wavelength spacing of the interference pattern can be expressed as

$$
\Delta \lambda=\frac{\lambda^{2}}{\Delta n \cdot L-\lambda \cdot L \cdot \frac{d \Delta n}{d \lambda}}
$$

where

$\Delta n \quad$ is the differential effective refractive index of core and cladding modes;

$L \quad$ is the distance between the two LPFG's;

$\lambda \quad$ is the center wavelength of the interference spectrum.

The fiber between the second LPFG and the fiber mirror is coiled to induce a large loss for the cladding mode and avoid other interference. To obtain an interference pattern with large

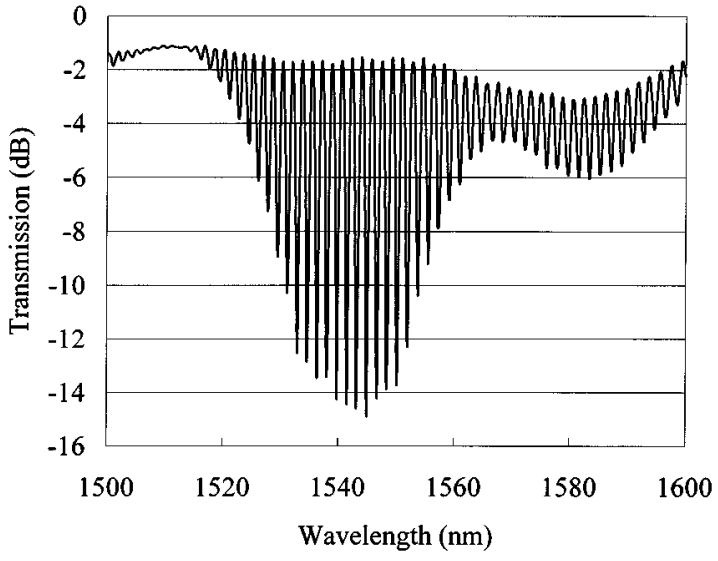

Fig. 2. Transmission spectrum of the cascaded LPFG's.

visibility or contrast, the power of core and cladding modes should be almost the same. Therefore, one should control the exposure dosage to obtain each LPFG with a peak loss of $3 \mathrm{~dB}$ so that $50 \%$ of light is coupled to the cladding and the other $50 \%$ remains in the core. Fig. 2 shows the transmission spectrum of the cascaded LPFG's. The period of the index variation and the length of the LPFG are chosen to be $400 \mu \mathrm{m}$ and $8 \mathrm{~mm}$, respectively, to have a loss band which covers the EDF's gain spectrum. The fiber length between the two LPFG's is $\sim 13 \mathrm{~cm}$ and the wavelength (channel) spacing of the interference pattern is $\sim 1.7 \mathrm{~nm}$. It is seen that a total of 14 loss peaks in the center of the LPFG's loss band can have a peak loss greater than 10 $\mathrm{dB}$. Since the interference pattern is sinusoidal, the $3-\mathrm{dB}$ width of each peak is half of the wavelength spacing, $0.85 \mathrm{~nm}$, and the finesse is only 2 .

It is shown that the characteristics of an SFS are configuration dependent. The DPB SFS can have a one-humped or two-humped spectrum with different EDF length, however, the DPF one can only have a one-humped spectrum. In the following, we choose different EDF lengths for the DPB and DPF MWFS's to characterize their properties. For the DPB configuration, the EDF lengths are chosen to be 10,15 , and $20 \mathrm{~m}$. Such lengths are chosen due to the following reasons. The $20-\mathrm{m}$ one has a pump-power-independent mean-wavelength operation. Both the 10- and 20-m DPB SFS have one-humped spectra. The hump of the former is centered at $\sim 1530 \mathrm{~nm}$ band and the latter at $1558 \mathrm{~nm}$ band. The $15-\mathrm{m}$ DPB SFS has a spectrum of two-humps centered at both bands. For a DPF SFS with larger output power, the spectrum with only one hump centered at 1530 $\mathrm{nm}$ band could be obtained. The 10-m EDF length is chosen such that the output power is the largest for the DPF SFS, and $15-\mathrm{m}$ length is chosen for comparison.

Fig. 3 shows the output spectra of DPB SFS's and their corresponding MWFS's for different EDF lengths pumped at 85 $\mathrm{mW}$. By inserting the cascaded LPFG's into each DPB SFS, the forward amplified spontaneous emission (ASE) signal filtered by the interference pattern is reflected by the fiber mirror. As the signal propagates through the EDF again, it will cause the ASE to redistribute according to the reflected and filtered signal. The greater the intensity in any spectrum range, the larger the ASE will be in that range so that the output spectrum of the proposed MWFS follows the interference pattern of the cascaded 


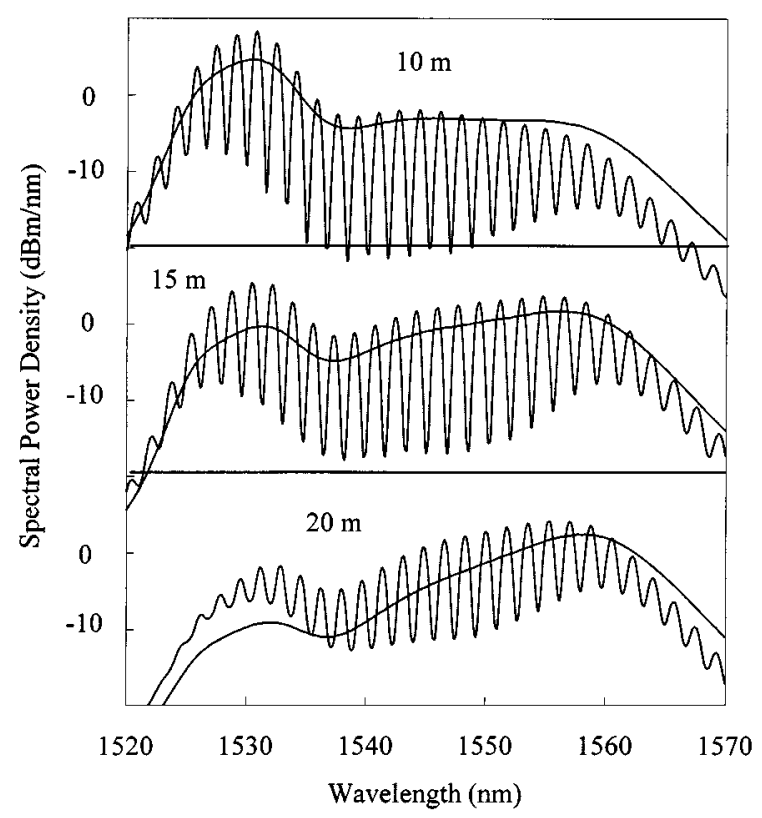

Fig. 3. Output spectra of SFS's and MWFS's based on DPB configuration.

LPFG's. Each peak of MWFS corresponds to the peak wavelength of the interference pattern. The channel spacing of such a source is also the same as the wavelength spacing of the interference pattern. Therefore, the channel spacing could be easily controlled by varying the distance between the two LPFG's or the effective refractive index difference between the core and cladding of different expose dosage. It is seen that the power level for several channels is larger than that of the original DPB SFS in the same wavelength. This is caused by the cascaded LPFG's, which not only filters the signal but also causes power redistribution. For a spectrum sliced source, a cascaded LPFG's or other filter is positioned at the output end of an SFS and only filters the ASE signal. Thus, the power of each channel is at most the same as that in the SFS if the insertion loss of the cascaded LPFG's is ignored. Therefore, the proposed MWFS could have more power in each channel than that of a spectrum sliced source over the wavelength range of interest, 1525-1560 $\mathrm{nm}$. Total output power are 24.7 and 32.9, 29.5, and 33.5, 24.8 and $23.1 \mathrm{~mW}$ for the MWFS and original DPB SFS with EDF lengths of 10,15 , and $20 \mathrm{~m}$, respectively. The power degradation by inserting the cascaded LPFG's into the DPB SFS is only 1.25, 0.55 , and $0.30 \mathrm{~dB}$, respectively. Due to the sinusoidal-shaped spectrum of cascaded LPFG's, the loss is at least $3 \mathrm{~dB}$ for any signals passing through. However, the cascaded LPFG's are positioned before the fiber mirror for the proposed MWFS rather than at the output end for a spectrum sliced source; therefore, only the weak forward ASE signal is filtered, which causes little power degradation. The power loss becomes smaller for a proposed MWFS having a longer EDF length because the forward ASE signal is weaker. In the following, we are interested in the channels between $1525-1560 \mathrm{~nm}$ due to the possibility of obtaining a large extinction ratio, e.g., more than $10 \mathrm{~dB}$. The extinction ratio can be used to evaluate the amount of power redistribution. A larger extinction ratio means more power redistribution. As the channel's power increase, it will lead to the reduction of crosstalk between adjacent channels. From Fig. 3, one can see that there are 20 channels with a larger extinction ratio near the center of LPFG's loss band for the MWFS's with 10and 15-m EDF's but only nine channels for that with 20-m EDF. For the MWFS's with 20-m EDF, the chosen pump power is not sufficient so that the signal has gains only in the front section of EDF but has losses in the rear section. The loss in the short wavelength is larger than that in the long wavelength, therefore, the spectrum of the forward ASE signal before passing the cascaded LPFG's is dominant at $1558 \mathrm{~nm}$ band. After filtered by the cascaded LPFG's and propagated through the EDF again, the power of channels at the $1558 \mathrm{~nm}$ band $(1540-1560 \mathrm{~nm})$ are larger than those in the $1530 \mathrm{~nm}$ band $(1525-1540 \mathrm{~nm})$. It is noted that the channel with the largest power is not centered at the peak wavelength $(\sim 1558 \mathrm{~nm})$ of the original DPB SFS due to the unequal peak transmittance of each channel of the cascaded LPFG's. The maximum extinction ratio of the 20-m DPB MWFS is $\sim 14 \mathrm{~dB}$ for the channel centered at $1550.1 \mathrm{~nm}$. For the MWFS with 10-m EDF, the chosen pump power is sufficient to provide gains for any section of EDF. Therefore, the spectrum is similar to the gain spectrum which is dominant at $1530 \mathrm{~nm}$ band. As the filtered ASE is reflected and propagates through the EDF again, the power of the channels near the $1530 \mathrm{~nm}$ dominant band is larger than the others. It is noted that the variation of extinction ratio among the channels between $1530-1550 \mathrm{~nm}$ is small, $\sim 2 \mathrm{~dB}$. The maximum extinction ratio is $19.6 \mathrm{~dB}$ at $1542.7 \mathrm{~nm}$. For the MWFS with $15-\mathrm{m}$ EDF, the power variation among channels having larger extinction ratios is smaller than those of the MWFS's with 10- or 20-m EDF's. This is because the original spectrum of $15-\mathrm{m}$ DPB SFS has two humps and is more flattened. The variation of extinction ratio of the channels between $1538-1555 \mathrm{~nm}$ is smaller than $3 \mathrm{~dB}$. The maximum extinction ratio is $19.9 \mathrm{~dB}$ at $1549.4 \mathrm{~nm}$.

From Fig. 3, the 10- and 15-m DPB MWFS could have more than 15 channels with large extinction ratios. We further study the effect of pump power on the DPB MWFS of these two lengths, and the results are shown in Fig. 4. Two channels, which together with all the channels in between have extinction ratios larger than $10 \mathrm{~dB}$, and another channel with the largest extinction ratio are selected. The channels are centered at 1525.8, 1556.7, and 1542.7 $\mathrm{nm}$ for the 10-m DPB MWFS and 1527.0, 1558.4, and 1549.4 $\mathrm{nm}$ for the 15-m DPB MWFS. It is shown that the power and extinction ratio increase with pump power. The extinction ratio becomes saturated as pump power increases. For the 10-m DPB MWFS, the required pump power is $30 \mathrm{~mW}$ so that all the channels between the selected channels have extinction ratios greater than $10 \mathrm{~dB}$, while the required power is $42 \mathrm{~mW}$ for the 15-m DPB MWFS. For multiwavelength generation, the forward ASE should propagate to the cascaded LPFG's. If the pump power is not sufficient or the EDF is too long, little forward ASE reaches the cascaded LPFG's and there is no multiwavelength generation. As the pump power increases, more forward signal is filtered by the cascaded LPFG's, and the extinction ratio increases. It is noted that for a longer EDF a larger pump power is required for multiwavelength generation in the DPB configuration.

Fig. 5 shows the output spectra of the DPF SFS and the MWFS based on the DPF configuration pumped at $85 \mathrm{~mW}$. As the cascaded LPFG's is inserted before the fiber mirror, 


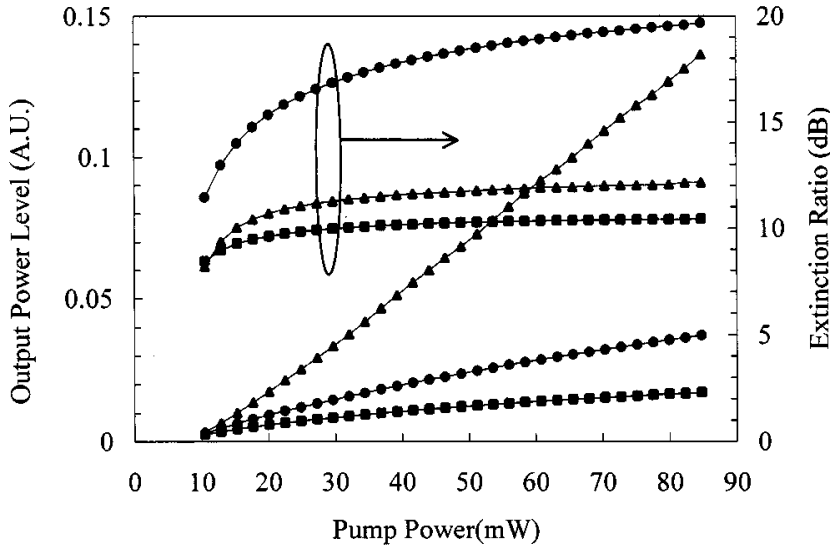

(a)

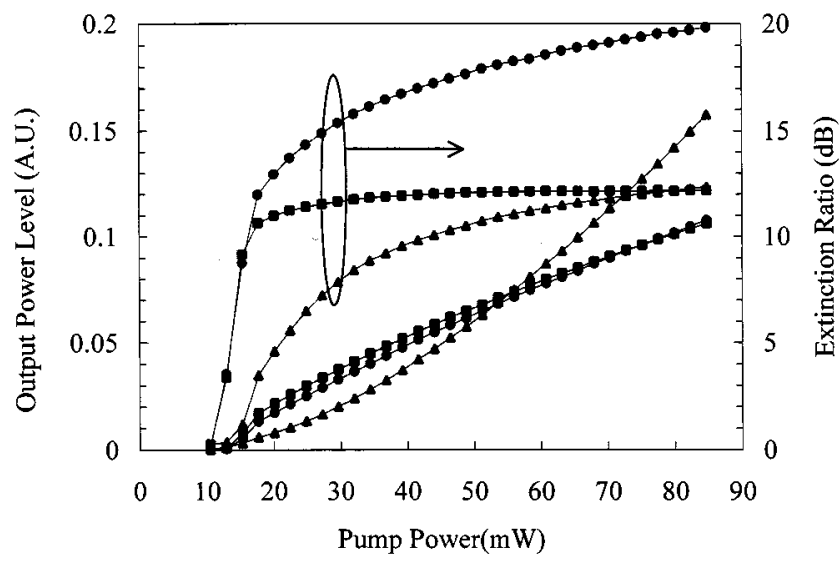

(b)

Fig. 4. Output power and extinction ratio versus pump power for (a) 10-m and (b) 15-m DPB MWFS's. The solid circles represent the channel having the largest extinction ratio. The solid triangle and rectangular represent the shortest and longest channels with an extinction ratio larger than $10 \mathrm{~dB}$, respectively.

similar to the mechanism described previously for DPB configuration, the output spectrum will have the same peaks as the interference pattern of the cascaded LPFG's. Due to the power redistribution by adding the cascaded LPFG's, there are several channels whose power level are greater than those in the original DPF SFS's. The output power of the DPF SFS and the corresponding MWFS are 25.0 and $24.5 \mathrm{~mW}, 19.5$ and $18.6 \mathrm{~mW}$ for both 10 - and $15-\mathrm{m}$ ones, respectively. The power degradation by inserting the cascaded LPFG's for these two MWFS's are only 0.1 and $0.2 \mathrm{~dB}$, which is also smaller than those in the spectrum sliced source. There are total 20 channels with extinction ratios larger than $10 \mathrm{~dB}$ for both $10-$ and $15-\mathrm{m}$ DPF MWFS's. The maximum extinction ratio is 20.1 and 18.1 $\mathrm{dB}$ for the 10- and 15-m DPF MWFS's, respectively. It is noted that the extinction ratio of the 10-m DPF MWFS is nearly the same, about $20 \mathrm{~dB}$, between 1530-1548 $\mathrm{nm}$.

Fig. 6 shows the effects of pump power on the characteristics of DPF MWFS's with EDF lengths of 10 and $15 \mathrm{~m}$. The channels are centered at $1525.8,1541.0$, and $1556.7 \mathrm{~nm}$ for the 10-m DPF MWFS and 1526.2, 1543.1, and $1557.1 \mathrm{~nm}$ for the 15-m MWFS. The power and extinction ratio for each channel also increase with pump power. The required pump power is

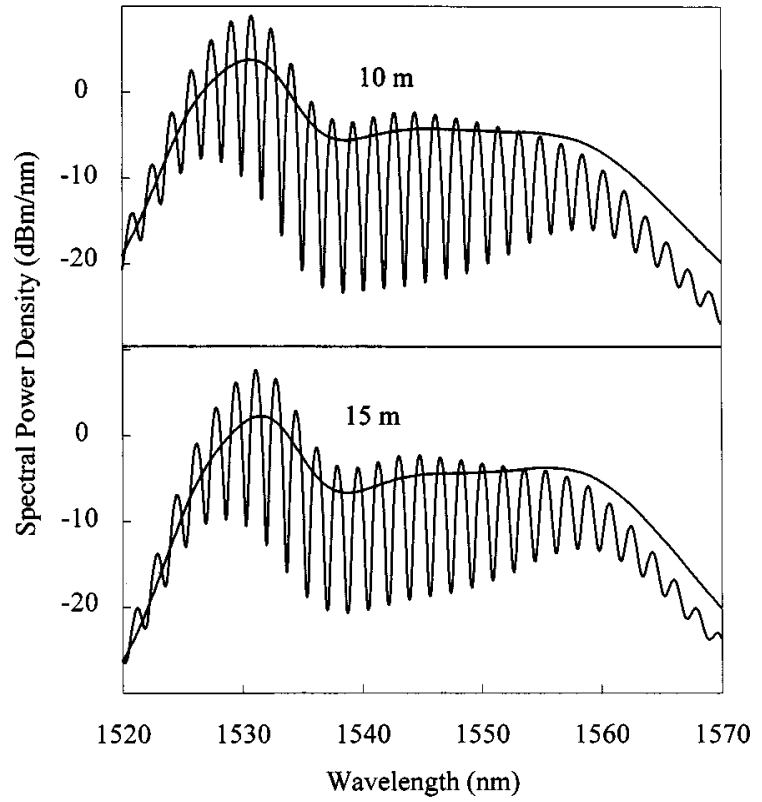

Fig. 5. Output spectra of SFS's and MWFS's based on DPF configuration.

only $18 \mathrm{~mW}$ for the $10-\mathrm{m}$ DPF MWFS and $20 \mathrm{~mW}$ for the $15-\mathrm{m}$ one such that all the channels between the selected channels have extinction ratios greater than $10 \mathrm{~dB}$. When the pump power is slightly greater than the threshold for the DPF MWFS with any EDF length, the backward ASE signal is always filtered by the cascaded LPFG's such that the MWFS could be generated. No matter how long the EDF is used, the required pump power for multi-wavelength generation is nearly the same. However, the pump power for the DPB MWFS should be sufficient to generate sufficient forward ASE signal, which can propagate through the cascaded LPFG's and be filtered. Thus, the longer the EDF is the larger the required pump power for the DPB MWFS is. Therefore, the required pump power to generate all the 20 channels with extinction ratios larger than $10 \mathrm{~dB}$ is smaller for the DPF MWFS than that for the DPB one with the same EDF length.

\section{DISCUSSION}

In the previous sections, we show that as a cascaded LPFG's is employed in the conventional double-pass SFS's to generate a MWFS, many channels can have greater power than the original double-pass SFS's due to power redistribution. The largest extinction ratio of the proposed MWFS is about $20 \mathrm{~dB}$, and could not be further increased because of residual single-pass ASE. This extinction ratio of the proposed MWFS is larger than that of the cascaded LPFG's spectrum. Therefore, the proposed MWFS will have larger power and extinction ratio in the interested channels than the spectrum sliced fiber source with the same cascaded LPFG's. Several spectrum sliced WDM systems have been demonstrated. A MWFS with extinction ratio of $6 \mathrm{~dB}$ was used to demonstrate a WDM system operating at $622 \mathrm{Mb} / \mathrm{s}$ [13]. Recently, Han et al. has demonstrated a $2.5-\mathrm{Gb} / \mathrm{s}$ spectrum sliced WDM system, where the extinction ratio mainly obtained by array waveguide grating is $\sim 25 \mathrm{~dB}$ [14]. As our proposed MWFS is to be used for a WDM system, each channel will be 


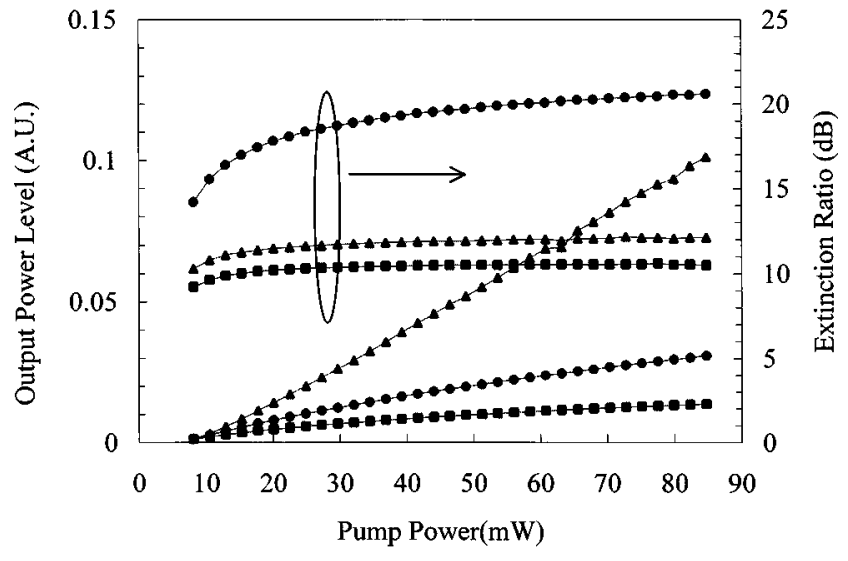

(a)

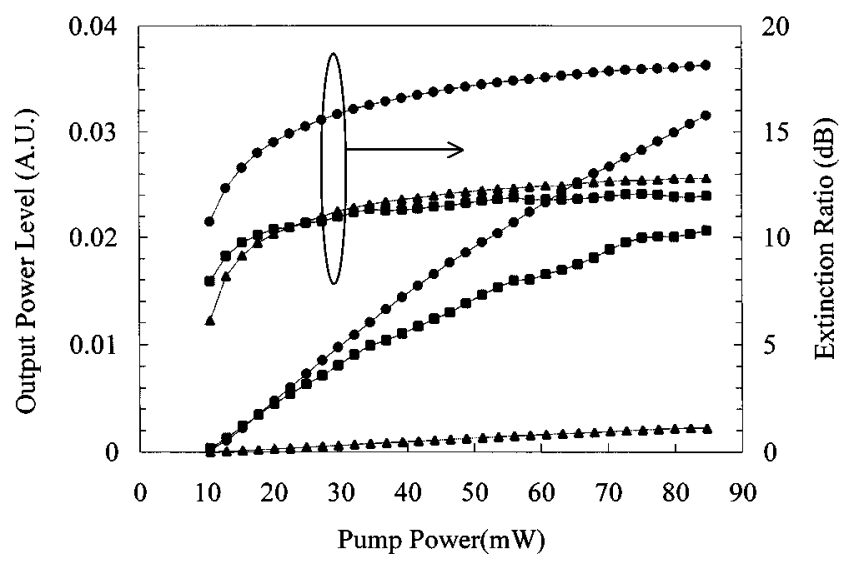

(b)

Fig. 6. Output power and extinction ratio versus pump power for: (a) 10-m and (b) 15-m DPF MWFS's. The solid circles represent the channel having the largest extinction ratio. The solid triangle and rectangular represent the shortest and longest channels with an extinction ratio larger than $10 \mathrm{~dB}$, respectively.

modulated, and a demultiplexer, e.g., array waveguide grating or DWDM, is needed to separate each channel. It has been indicated that the crosstalk of a spectrum-sliced WDM system is contributed from the out-of-band rejection level of the demultiplexer [15]. Therefore, we expect that the proposed MWFS can be a candidate source for Gigabits WDM system when a demultiplexer with large out-of-band rejection level is used for crosstalk reduction.

It is noted that the envelopes of channel's power level basically follow the original spectra of the double-pass SFS's. Only the 15-m MWFS based on DPB configuration could have channels with power variation less than $10 \mathrm{~dB}$ in the wavelength range of interest. For other cases, most power is centered in the dominant bands of the original double-pass SFS's, which will limit the application of such a MWFS. To decrease the power variation among channels, an original double-pass SFS with more flattened spectrum is required. It has been shown that by inserting an appropriate LPFG before the fiber mirror the spectrum of a DPB SFS could be broadened [16]. Therefore, we insert another LPFG named LPFG1 between the WDM and the cascaded LPFG's, shown in Fig. 7, to obtain a flattened MWFS. The fiber between LPFG1 and the cascaded LPFG's should also

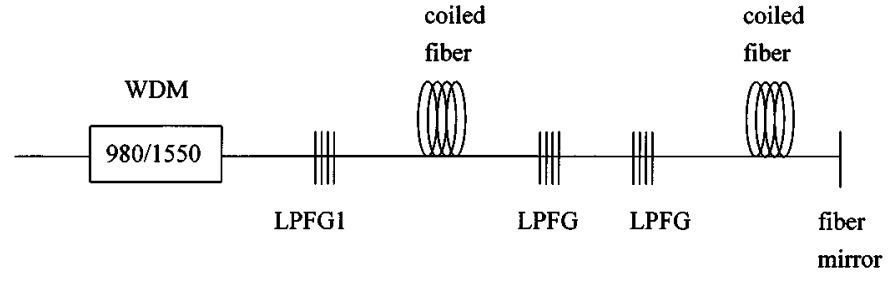

Fig. 7. Schematic diagram of a flattened MWFS.

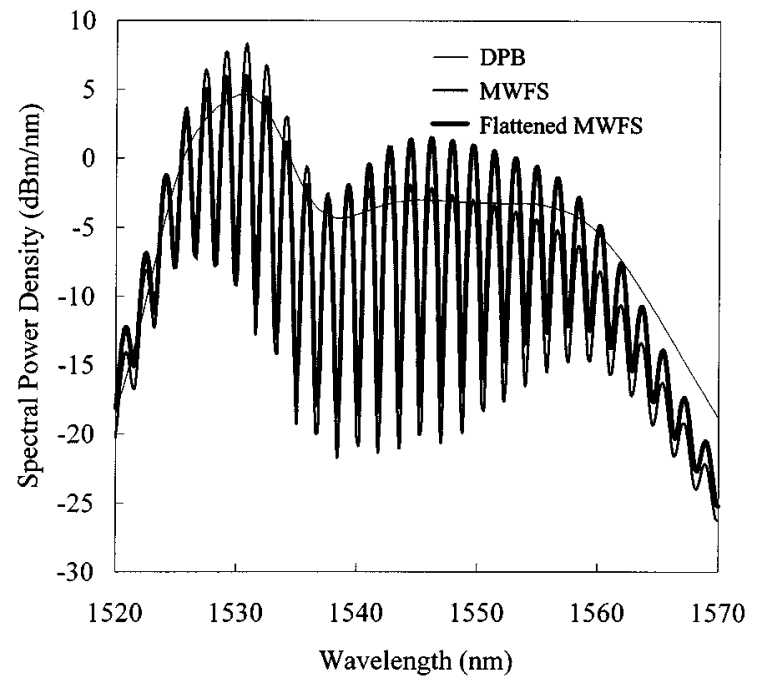

Fig. 8. Output spectra of a 10-m flattened DPB MWFS.

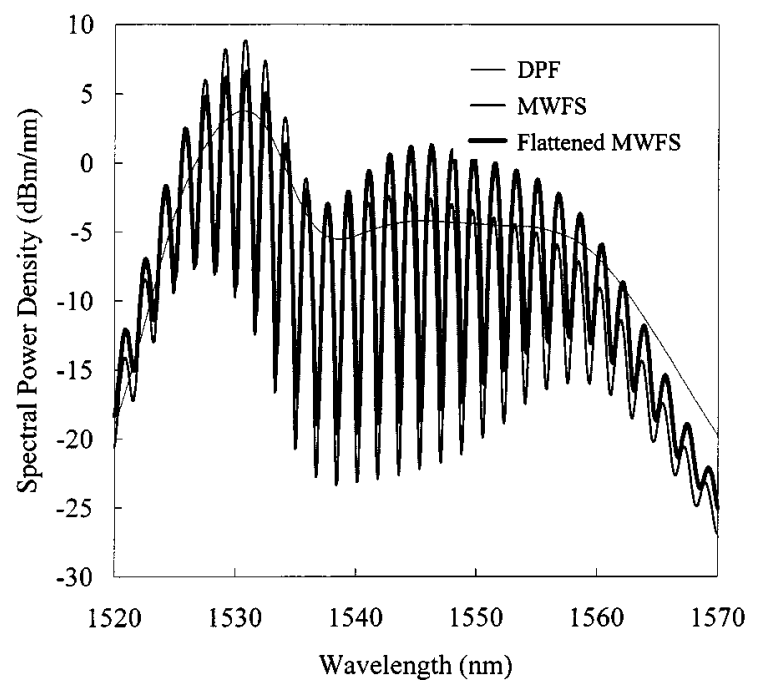

Fig. 9. Output spectra of a 10-m flattened DPF MWFS.

be coiled to avoid formation of another interference pattern. Fig. 8 and 9 show the effect of adding LPFG1 on the 10-m MWFS in the DPB and DPF configuration, respectively. The LPFG1 has a peak loss of $6 \mathrm{~dB}$ centered at $1530.2 \mathrm{~nm}$ and has a 3 -dB width of $14.8 \mathrm{~nm}$. It is clearly seen that power of the channels near the $1530 \mathrm{~nm}$ band decreases while that of the channels outside the band increases. As power of channels outside the LPFG1's loss band increases, the residual single pass ASE also increases. Therefore, the extinction ratio remains nearly the same after inserting LPFG1 to flatten the MWFS. The power 


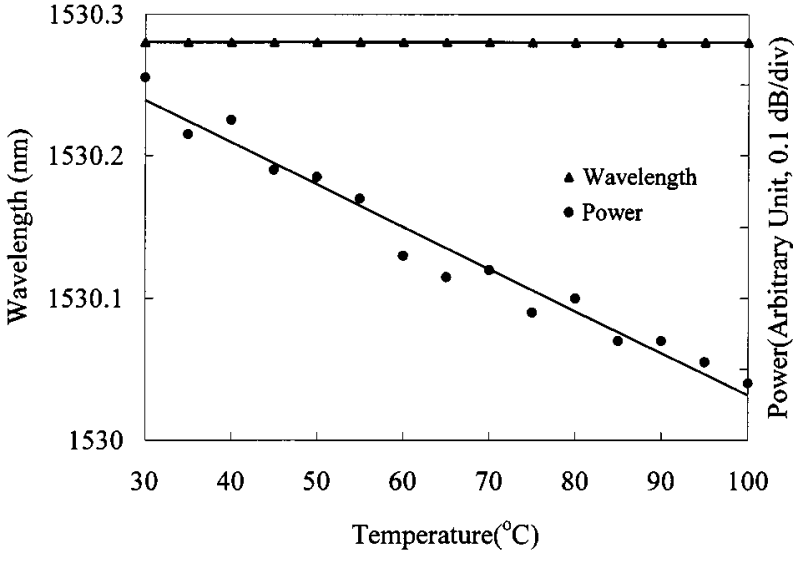

Fig. 10. Temperature effect of EDF on the stabiltiy of a MWFS.

variation among channels decreases from 15 to $8 \mathrm{~dB}$ for the 10-m DPB MWFS and 16 to $9 \mathrm{~dB}$ for the 10-m DPF one. If a more appropriated LPFG is utilized, a MWFS with nearly the same power in each channel can be generated.

For WDM applications, each channel of a MWFS should have stable wavelength and power. In the following, we discuss the temperature stability of the proposed MWFS, including the temperature effects of EDF and cascaded LPFG's. The 10-m MWFS in DPF configuration is taken as an example. To distinguish temperature effects of EDF and cascaded LPFG's, the temperature of the EDF and cascaded LPFG's is controlled separately from room temperature to $100{ }^{\circ} \mathrm{C}$ while the temperature of the other components is set at room temperature. Fig. 10 shows the temperature effect of EDF on the MWFS. The channel centered at $1530.3 \mathrm{~nm}$ is selected for instance. The peak wavelength remains the same although the temperature of EDF varies from room temperature to $100{ }^{\circ} \mathrm{C}$. As the temperature of EDF increases, the power level decreases slightly because the gain spectrum shifts slightly toward long wavelength side as temperature increases. The power variation rate is only $0.006 \mathrm{~dB} /{ }^{\circ} \mathrm{C}$.

Fig. 11 shows the temperature effect of cascaded LPFG's on the MWFS. As the temperature of LPFG's increases, the peak wavelength shifts toward long wavelength side at a rate of 0.016 $\mathrm{nm} /{ }^{\circ} \mathrm{C}$, resulted from the increased optical path difference of core and cladding modes. The thermal induced wavelength-shift of the cascaded LPFG's can be expressed as

$$
\frac{d \lambda}{d T}=\frac{L \cdot \Delta \lambda}{\lambda}\left(\frac{\partial \Delta n}{\partial T}+\Delta n \cdot \frac{1}{L} \frac{d L}{d T}\right) .
$$

$\Delta n$ can be calculated to be 0.00386 from the phase-matching condition of the LPFG. The thermal expansion coefficient of silica fiber is $\sim 5 \times 10^{-7} /{ }^{\circ} \mathrm{C}$ [17]. The wavelength-shift due to the thermal expansion is $0.000276 \mathrm{~nm} /{ }^{\circ} \mathrm{C}$, which is only $1.7 \%$ of the total shift. Therefore, the thermal induced wavelength-shift of the cascaded LPFG's is mainly contributed from the thermal variation of the differential effective index of the core and cladding mode. $(\partial \Delta n / \partial T)$ is calculated to be $1.1 \times$ $10^{-7}$. The result is consistent with that shown in [18]. The power level decreases slowly with the temperature of cascaded LPFG's at a rate of $0.008 \mathrm{~dB} /{ }^{\circ} \mathrm{C}$. Since the loss band of an LPFG shifts toward the long wavelength side with temperature, the contrast

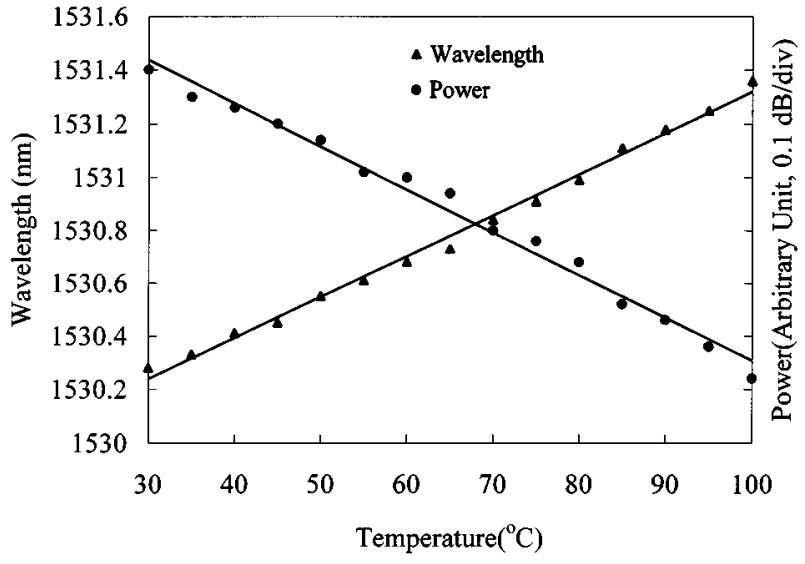

Fig. 11. Temperature effect of cascaded LPFG's on the stability of a MWFS.

of the channel becomes smaller than the original value, and results in a decrease in power level. From the above discussion, it can be concluded that the stability of the MWFS is mainly determined by the cascaded LPFG's.

\section{CONCLUSION}

We have demonstrated a new method to generate a high power MWFS by inserting cascaded LPFG's in a conventional double-pass SFS. For the MWFS in a DPB or DPF configuration, 20 channels with extinction ratios greater than $10 \mathrm{~dB}$ can be obtained between $1525-1560 \mathrm{~nm}$. The channel spacing and the 3-dB width of each channel are determined by the cascaded LPFG's. The channel spacing is the same as that of the interference pattern of the cascaded LPFG's. The 3-dB width is half of the channel spacing and thus the finesse is 2 . It is shown that the cascaded LPFG's not only provides the filtering effect but also causes the spectral power to redistribute. Therefore, the output power of each channel of the MWFS is larger than that of a spectrum sliced source. The characteristics of the proposed MWFS are configuration dependent. The required EDF length and pump power for generating MWFS are smaller in DPF configuration than those in DPB configuration. The total output power of the DPB MWFS is larger than that of DPF one. Little power degradation, e.g., smaller than $0.5 \mathrm{~dB}$, incurs when the cascaded LPFG's is used for both the DPB and DPF MWFS's.

The envelope of channel's power level follows the original spectrum of the double-pass SFS. Only the DPB MWFS with an appropriate EDF length, e.g., $15 \mathrm{~m}$ in our case, could have channels with smaller power variation. For DPF configuration or DPB one with other EDF lengths, the spectrum flattening is needed to obtain a MWFS with small channel's power variation. We propose a method by inserting another LPFG between the cascaded LPFG's and WDM for generation a MWFS with small channel's power variation. The results show that the power variation could be decreased from more than $15 \mathrm{~dB}$ to smaller than $10 \mathrm{~dB}$. It is possible that a more flattened MWFS could be obtained if a more appropriate LPFG is used. The measurement of temperature stability of MWFS shows that the wavelength of each channel remains constant when the temperature of EDF increases, but increases at a rate of $0.016 \mathrm{~nm} /{ }^{\circ} \mathrm{C}$ as the temperature of cascaded LPFG's increases. The power variation rates 
with temperature of EDF and cascaded LPFG's are 0.006 and $0.008 \mathrm{~dB} /{ }^{\circ} \mathrm{C}$, respectively. Therefore, the stability of the proposed MWFS is mainly determined by the cascaded LPFG's rather than by the EDF.

\section{REFERENCES}

[1] M. C. Farries, A. C. Carter, G. G. Jones, and I. Bennion, "Tunable multiwavelength semiconductor laser with single fiber output," Electron. Lett., vol. 27, pp. 1498-1499, 1991.

[2] A. Hamakawa, T. Kato, G. Sasaki, and M. Shigehara, "A four-channel multiwavelength fiber-grating external cavity-laser array," in Proc. Optic. Fiber Commun. Conf., Tech. Dig., 1997, Paper ThM3.

[3] M. H. Reeve, A. R. Hunwicks, W. Zhao, S. G. Methley, L. Bickers, and S. Hornung, "LED spectral slicing for single-mode local loop applications," Electron. Lett., vol. 24, pp. 389-390, 1988.

[4] S. S. Wagner and T. E. Chapuran, "Broadband high-density WDM transmission using superluminescent diodes," Electron. Lett., vol. 26, pp. 696-697, 1990

[5] J. S. Lee, Y. C. Chung, and D. J. DiGiovanni, "Spectrum- sliced fiber amplifier light source for multichannel WDM applications," IEEE Photon. Technol. Lett., vol. 5, pp. 1458-1461, 1993.

[6] J. S. Lee, Y. C. Chuang, and C. S. Shim, "Bandwidth optimization of a spectrum-sliced fiber amplifier light source using an angle-tuned Fabry-Perot filter and a double-stage structure," IEEE Photon. Technol. Lett., vol. 6, pp. 1197-1199, 1994.

[7] D. K. Jung, S. K. Shin, C. H. Lee, and Y. C. Chung, "Wavelength-division-multiplexed passive optical network based on spectrum-slicing techniques," IEEE Photon. Technol. Lett., vol. 10, pp. 1334-1336, 1998.

[8] P. F. Wysocki, M. J. F. Digonnet, B. Y. Kim, and H. J. Shaw, "Characteristics of erbium-doped superfluorescent fiber sources for interferometric sensor applications," J. Lightwave Technol., vol. 12, pp. 550-567, 1994.

[9] X. J. Gu, "Wavelength-division multiplexing fiber filter and light source using cascaded long-period fiber gratings," Opt. Lett., vol. 23, pp. 509-510, 1998

[10] C. D. Su and L. A. Wang, "Multiwavelength fiber source by using long period fiber gratings in superfluorescent fiber source," Electron. Lett., pp. 927-929, 1999 .
[11] L. A. Wang and C.D. Chen, "Comparison of efficiency and output power of optimal Er-doped superfluorescent fiber sources in different configurations," Electron. Lett., vol. 33, pp. 703-704, 1997.

[12] A. M. Vengsarkar, P. J. Lemaire, J. B. Judkins, V. Bhatia, T. Erdogan, and J. E. Sipe, "Long-period fiber gratings as band-rejection filters," $J$. Lightwave Technol., vol. 14, pp. 58-65, 1996.

[13] W. T. Holloway, A. J. Keating, and D. D. Sampson, "Multiwavelength source for spectrum-sliced WDM Access Networks and LAN's," IEEE Photon. Technol. Lett., vol. 9, pp. 1014-1016, 1997.

[14] J. H. Han, S. J. Kim, and J. S. Lee, "Transmission of $4 \times 2.5-\mathrm{Gb} / \mathrm{s}$ spectrum-sliced incoherent light channels over $240 \mathrm{~km}$ of dispersionshifted fiber with 200-GHz channel spacing," IEEE Photon. Technol. Lett., vol. 11, pp. 901-903, 1999.

[15] R. D. Feldman, "Crosstalk and loss in wavelength division multiplexed systems employing spectral slicing," J. Lightwave Technol., vol. 15, pp. 1823-1831, 1997.

[16] C. D. Su and L. A. Wang, "Effect of adding a long period grating in a double-pass backward Er-doped superfluorescent fiber source," J. Lightwave Technol., vol. 17, pp. 1896-1903, 1999.

[17] G. B. Hocker, "Fiber optic sensing of temperature and pressure," Appl. Opt., vol. 18, pp. 1445-1448, 1979.

[18] B. H. Lee and J. Nishii, "Self-interference of long-period fiber grating and its application as temperature sensor," Electron. Lett., vol. 34, pp. 2059-2060, 1998.

C. D. Su, photograph and biography not available at the time of publication.

Lon A. Wang (M'95-A'96), photograph and biography not available at the time of publication. 